\title{
Long non-coding RNA regulates hair follicle stem cell proliferation and differentiation through PI3K/AKT signal pathway
}

\author{
BINGJIE CAI ${ }^{1}$, YUNPENG ZHENG ${ }^{1}$, SHANSHAN MA ${ }^{2}$, QU XING $^{2}$, XINXIN WANG $^{1}$, \\ BO YANG ${ }^{1}$, GUANGWEN YIN ${ }^{1}$ and FANGXIA GUAN ${ }^{1,2}$ \\ ${ }^{1}$ Department of Dermatology, The First Affiliated Hospital of Zhengzhou University, Zhengzhou, \\ Henan 450052; ${ }^{2}$ School of Life Sciences, Zhengzhou University, Zhengzhou, Henan 450001, P.R. China
}

Received August 14, 2017; Accepted December 15, 2017

DOI: $10.3892 / \mathrm{mmr} .2018 .8546$

\begin{abstract}
Long non-coding RNAs (lncRNAs) are defined as non-coding transcripts ( $>200$ nucleotides) that serve important roles in the proliferation and differentiation of stem cells. Hair follicle stem cells (HFTs) have multidirectional differentiation potential and are able to differentiate into skin, hair follicles and sebaceous glands, serving a role in skin wound healing. The aim of the present study was to analyze the regulatory role of IncRNA AK015322 (IncRNA5322) in HFTs and the potential mechanism of IncRNA5322-mediated differentiation of HFTs. The results demonstrated that lncRNA5322 transfection promoted proliferation and differentiation in HFTs. It was identified that IncRNA5322 transfection upregulated the expression and phosphorylation of phosphoinositide 3-kinase (PI3K) and protein kinase B (AKT) in HFTs. It was also observed that lncRNA5322 transfection upregulated microRNA (miR)-21 and miR-21 agonist (agomir-21) eliminated lncRNA5322-induced expression and phosphorylation of PI3K and AKT. The present study also demonstrated that agomir-21 blocked IncRNA5322-induced expression and phosphorylation of PI3K and AKT in HFTs. The results indicated that agomir-21 transfection also suppressed the IncRNA5322-induced proliferation and differentiation of HFTs. In conclusion, the results of the present study suggest that IncRNA5322 is able to promote the proliferation and
\end{abstract}

Correspondence to: Professor Guangwen Yin, Department of Dermatology, The First Affiliated Hospital of Zhengzhou University, 1 Jianshedong Road, Zhengzhou, Henan 450052, P.R. China E-mail: guangwenyinprof@163.com

Professor Fangxia Guan, School of Life Sciences, Zhengzhou University, 100 Kexueda Road, Zhengzhou, Henan 450001, P.R. China E-mail: guanfangxiasci@sina.com; guanfangxia@126.com

Key words: long non-coding RNA5322, proliferation, differentiation, hair follicle stem cells, microRNA-21, phosphoinositide 3-kinase, protein kinase $\mathrm{B}$ differentiation of HFTs by targeting the miR-21-mediated PI3K-AKT signaling pathway in HFTs.

\section{Introduction}

Long non-coding RNAs (lncRNA) are a class of small non-coding transcripts $>200$ nucleotides and have been identified to serve a role in the proliferation and differentiation of cells (1-3). In recent years, lncRNAs have become the focus of research into a number of human diseases, including metabolic and hereditary diseases, cancer and human stem cell differentiation (4-6). Evidence has indicated that lncRNA is associated with cellular signal pathway transduction, which suggests that lncRNA may integrate into the pluripotency network and be a target for patient-specific cell-based therapies (7-9). Molecular signaling mechanisms have confirmed that lncRNA is associated with a variety of cellular metabolism processes via regulating different signal pathways in human cells (HFTs) $(10,11)$.

HFTs are adult stem cells and have a marked proliferation ability in the skin wound healing process $(12,13)$. An immunohistochemical study suggested that HFTs are able to induce hair follicle growth by targeting Wnt10b (14). Shen et al (15) demonstrated that $\beta$-catenin induces HFT differentiation into transit-amplifying cells via upregulating c-myc activation. Another study indicated that the in vivo transcriptional governance of HFTs may be regulated by Wnt regulators (16). miR-128 is reported to regulate the differentiation of hair follicle mesenchymal stem cells into smooth muscle cells by targeting SMAD family member 2 (17). These reports suggest that HFTs may regulate cellular metabolism by regulating different molecule-mediated signal pathways.

The aim of the present study was to investigate the regulatory role of lncRNA AK015322 (IncRNA5322), which is regarded to be an important lncRNA for stem cells proliferation and differentiation (18), in the differentiation of HFTs. The results revealed the importance of the lncRNA5322/microRNA (miR)-21/phosphoinositide 3-kinase (PI3K)/protein kinase B (AKT) signaling pathway in the proliferation and differentiation of HFTs. It was also demonstrated that the proliferation and differentiation of hair follicle stem cells is based on the interaction between IncRNA5322 and miR-21, thereby regulating the PI3K/AKT signaling pathway in HFTs. 


\section{Materials and methods}

Cells and reagents. HFTs were purchased from Beijing Jing-Meng High-Tech Stem Cell Technology Co., Ltd. (Beijing, China). They were cultured in Minimum Essential Medium (MEM) (Sigma Aldrich; Merck KGaA, Darmstadt, Germany) supplemented with $10 \%$ fetal bovine serum (FBS; Invitrogen; Thermo Fisher Scientific, Inc., Waltham, MA, USA). All cells were cultured at $37^{\circ} \mathrm{C}$ in a humidified atmosphere containing $5 \% \mathrm{CO}_{2}$.

Transfection of lncRNA5322 or miR-21 assay. LncRNA5322 (19) or miR-21 (5'-UCAACAUCAGUCAGA UAAGCUA-3') and negative control lncRNA-vector (18) or miR-vector (control, 5'-UAGCUUAUCAGACAGAUGU UGA-3') were obtained from Shanghai GenePharma Co., Ltd. (Shanghai, USA). plncRNA5322 or pmiR-21 was cloned into the pBabe vector (Cell Biolabs, Inc., San Diego, CA, USA) to generate the plncRNA5322 and pmiR-21 vectors. Transfection of plncRNA5322, pmiR-21 and negative control vectors was performed using X-treme GENE RNA transfection reagent (Roche Applied Science, Rotkreuz, Switzerland). Transfection concentrations were $100 \mathrm{nM}$ for plncRNA5322 and pmiR-21 or negative vector. After 48 -h following transfection, cells were used to further analysis.

Cells proliferation and differentiation. HFTs were cultured and treated with agomir-21 or PI3K inhibitor $(0.5 \mathrm{mg} / \mathrm{ml}$, Guangzhou RiboBio Co., Ltd., Guangzhou, China) at $37^{\circ} \mathrm{C}$ in a humidified atmosphere containing $5 \% \mathrm{CO}_{2}$. Cell proliferation and differentiation were analyzed as previously described (20,21). For cell differentiation, HFTs were cultured in MEM for $12 \mathrm{~h}$ at $37^{\circ} \mathrm{C}$. HFT colonies growing on Matrigel (Corning China, Ltd., Shanghai, China) were loosely detached by dispase treatment for $5 \mathrm{~min}$ and washed 3 times with PBS. Cells were resuspended in Dulbecco's modified Eagle's medium (Sigma Aldrich; Merck KGaA) containing 20\% FBS. Cells were maintained on $1 \%$ agar-coated slides and allowed to differentiate for another 18 days at $37^{\circ} \mathrm{C}$. Cells were subsequently fixed with $10 \%$ formalin for $1 \mathrm{~h}$ at $37^{\circ} \mathrm{C}$ and stained with $60 \%$ Oil Red O in isopropanol as working solution for $10 \mathrm{~min}$ at $37^{\circ} \mathrm{C}$. The proportion of Oil Red O-positive cells was determined by counting stained cells under a light microscope at 40x magnification. For cell proliferation assay, HFTs were seeded in 96-well plates $\left(10^{3}\right.$ cells/well) and cultured for $24 \mathrm{~h}$ at $37^{\circ} \mathrm{C}$. Cells proliferation was determined using a Cell Counting Kit-8 (Dojindo Molecular Technologies, Inc., Kumamoto, Japan) according to the manufacturer's protocol.

Reverse transcription-quantitative polymerase chain reaction (RT-qPCR). Total RNA was extracted from HFTs using the RNAeasy Mini kit (Qiagen Sciences, Inc., Gaithersburg, MD, USA) and $1 \mu \mathrm{g}$ total RNA was transcribed into cDNA using an RT kit (Qiagen Sciences, Inc.) for $1.5 \mathrm{~h}$ at $42^{\circ} \mathrm{C}$. The cDNA (10 ng) was subjected to a qPCR using a SYBR-Green Master Mix system (Bio-Rad Laboratories, Inc., Hercules, CA, USA). All primers were synthesized by Invitrogen (Thermo Fisher Scientific, Inc.) and sequences are as follows: Cyclin-dependent kinase (CDK)1 forward, 5'-CAGACTAGA AAGTGAAGAGGAAGG-3' and reverse, 5'-ACTGACCAG
GAGGGATAGAATC-3'; CDK2 forward, 5'-TTGGCAGCA CACTCTATG-3' and reverse, 5'-CCTCATTCGGCAAAT AAACG-3'; LncRNA5322 forward, 5'-GACGAACTGACC GGTTGTCT-3' and reverse, 5'-GTGACAGAGGGATAG CGAGC-3'; and $\beta$-actin forward, 5'-CGGAGTCAACGG ATTTGGTC-3' and reverse, 5'-AGCCTTCTCCATGGTCG TGA- 3 '. The following thermocycling conditions were applied: 45 amplification cycles consisting of denaturation at $95^{\circ} \mathrm{C}$ for $30 \mathrm{sec}$, primer annealing at $63^{\circ} \mathrm{C}$ for $45 \mathrm{sec}$ with touchdown to $57^{\circ} \mathrm{C}$ for $50 \mathrm{sec}$, and applicant extension at $72^{\circ} \mathrm{C}$ for $60 \mathrm{sec}$. Relative mRNA expression changes were calculated using the $2^{-\Delta \Delta C q}$ method (22). The results are expressed as the $n$-fold way compared to control.

Western blotting. HFTs were collected and lysed in radioimmunoprecipitation assay buffer (mammalian protein extraction reagent for the cells and tissue protein extraction reagent for the tissues; Thermo Fisher Scientific, Inc.) followed by homogenization at $4^{\circ} \mathrm{C}$ for $10 \mathrm{~min}$. Protein concentration was measured by a Biconchoninic Acid protein assay kit (Thermo Fisher Scientific, Inc). A total of $20 \mu \mathrm{g}$ protein/lane was separated by $12.5 \%$ SDS-PAGE and transferred onto nitrocellulose membranes. The membranes were incubated in blocking buffer (5\% milk) for $2 \mathrm{~h}$ at $37^{\circ} \mathrm{C}$ prior to incubation with primary antibodies at $4^{\circ} \mathrm{C}$ overnight. The primary rabbit anti-mouse antibodies used in the present study were: CDK1 (ab133327; 1:1,000; Abcam, Cambridge, UK), CDK2 (ab76146; 1:1,000; Abcam), PI3K (ab182651; 1:200; Abcam), AKT (ab8805; 1:1,000; Abcam), phosphorylated (p)PI3K (ab182651; 1:1,000; Abcam), pAKT (ab38449; 1:500; Abcam) and $\beta$-actin (ab8226; 1:500; Abcam). A horseradish peroxidase-conjugated anti-rabbit IgG (1:5,000; Bio-Rad Laboratories, Inc.) was used as the secondary antibody. Bands were visualized using Western Blotting Luminol Reagent (Pierce ${ }^{\mathrm{TM}}$ Fast Western

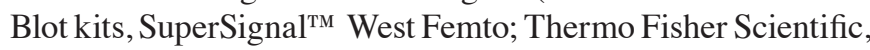
Inc.). Bands intensities normalized to $\beta$-actin. The density of the bands was analyzed by Quantity One software version 4.62 (Bio-Rad Laboratories, Inc.).

Luciferase reporter assay. The 3'-untranslated region (3'-UTR) sequence of PI3K and AKT, predicted to interact with lncRNA AK015322 or IncRNA vector (control) sequence within the predicted target sites (http://www.cbcb.umd.edu/software/GeneSplicer/gene_spl.shtml), was inserted into the pGL3 control vector (Promega Corporation, Madison, WI, USA). These constructs were designated as PI3K-3'-UTR and AKT-3'-UTR, respectively. For the reporter assay, HFTs cells were seeded in 24-well plates and transfected with the above constructs using Lipofectamine ${ }^{\circledR} 3000$ (Thermo Fisher Scientific, Inc.), IncRNA AK015322 expression vector and negative control. After $48 \mathrm{~h}$, the cells were collected and Renilla luciferase activity was measured using the Dual-Luciferase Reporter Assay System (Promega Corporation) according to the manufacturer's protocols. Results were obtained from 3 independent experiments performed in duplicate.

Statistical analysis. Data are presented as the mean \pm standard deviation of triplicate dependent experiments and analyzed using Student t-tests or one-way analysis of variance followed by Tukey's honest significant difference test. 
A
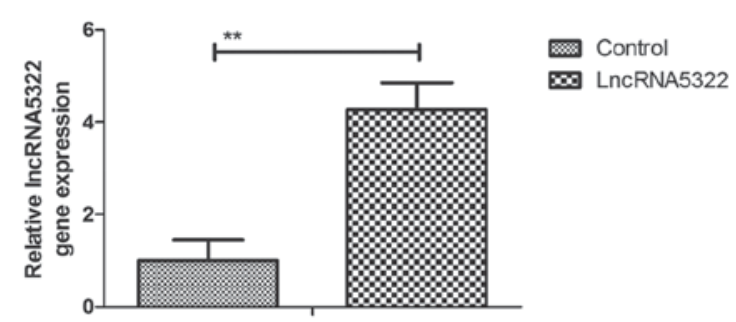

C

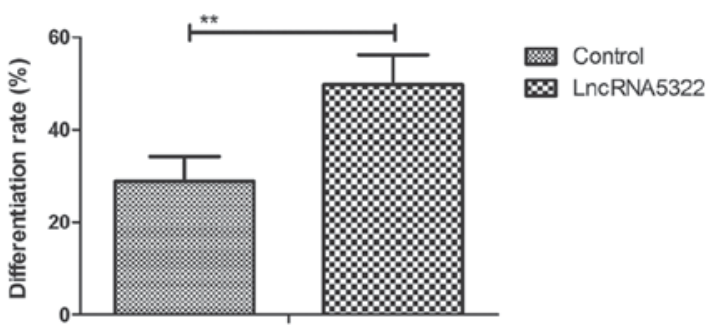

B

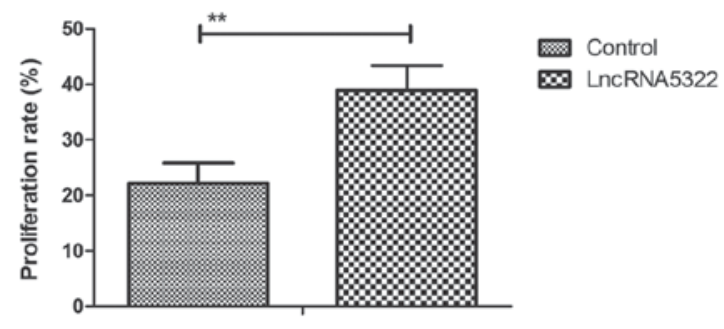

Figure 1. LncRNA5322 promotes the proliferation and differentiation of HFTs. Transfection with 1ncRNA5322 (A) increases 1 ncRNA5322 expression (B) stimulates proliferation and (C) promotes differentiation in HFTs. ${ }^{* *} \mathrm{P}<0.01$. Lnc, long non-coding; HFT, hair follicle stem cell.

A

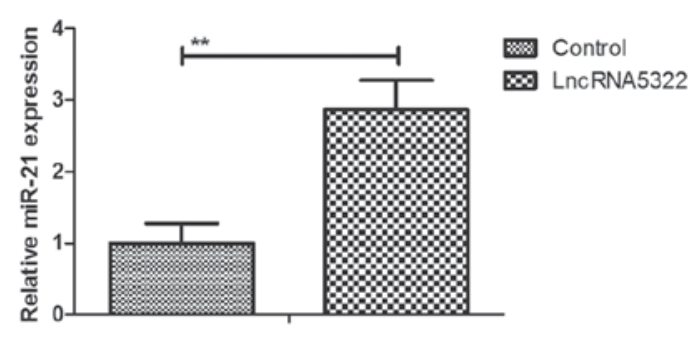

C



B

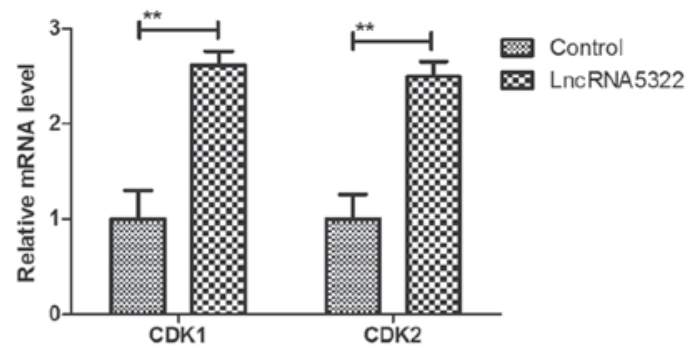

D

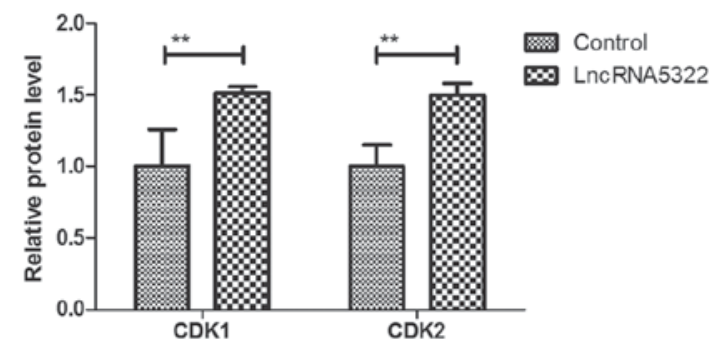

Figure 2. LncRNA5322 upregulates miR-21 expression and cell cycle during HFT differentiation. LncRNA5322 transfection upregulates (A) miR-21 expression as well as CDK1 and CDK2 (B) mRNA and (C) protein expression in HFTs. Band intensities were normalized to $\beta$-actin. (D) Effects of LncRNA5322 transfection on CDK1 and CDK2 expression in HFTs. ${ }^{* *} \mathrm{P}<0.01$. Lnc, long non-coding; miR, microRNA; HFT, hair follicle stem cell; CDK, cyclin-dependent kinase.

Significance was established using SPSS 17.0 (SPSS, Inc., Chicago, IL, USA) and GraphPad Prism 5 software (GraphPad Software, Inc., La Jolla, CA, USA). P $<0.05$ was considered to indicate a statistically significant difference.

\section{Results}

LncRNA5322 stimulates proliferation and differentiation of HFTs. The effect of IncRNA5322 on the proliferation and differentiation of HFTs was investigated. Transfection with lncRNA5322 increased lncRNA5322 expression in HFTs compared with the control (Fig. 1A). The results demonstrated that IncRNA5322 transfection stimulated proliferation of HFTs compared with control cells (Fig. 1B). The results also revealed that PlncRNA-1 transfection markedly promoted HFT differentiation (Fig. 1C). These results indicate that lncRNA5322 transfection induces the proliferation and differentiation of HFTs. 
A

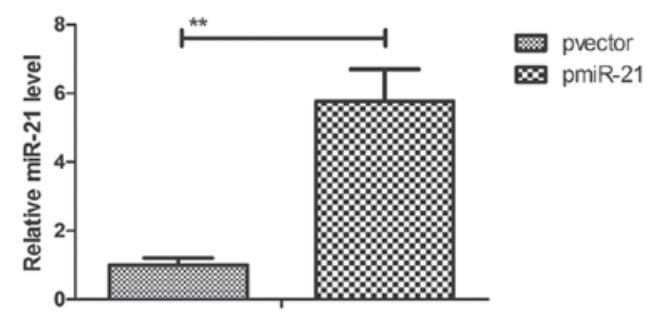

C



B

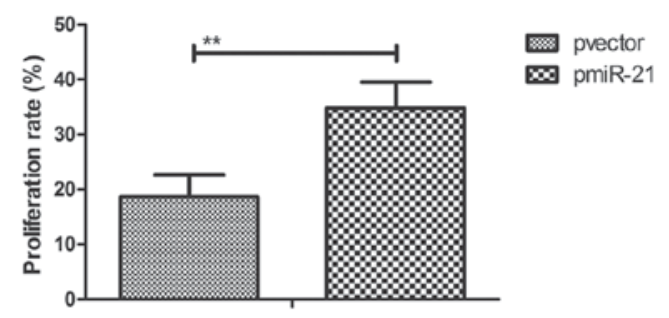

D

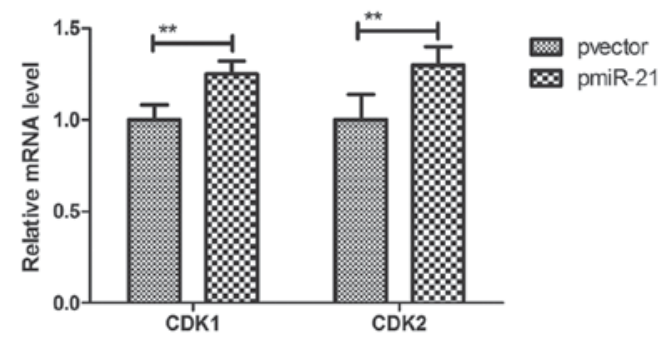



$\mathbf{F}$

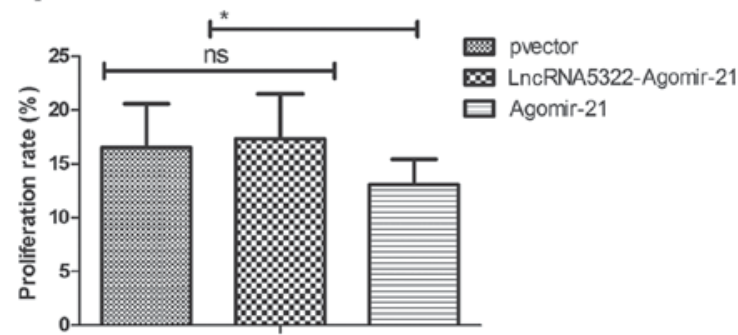

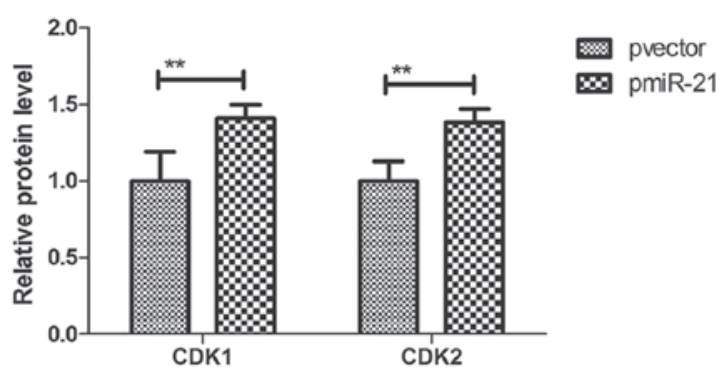

G

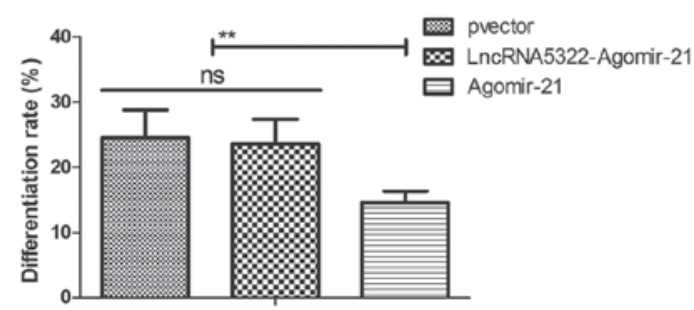

Figure 3. Transfection with miR-21 promotes HFT proliferation and differentiation. Transfection with miR-21 increases (A) miR-21 expression, (B) proliferation and (C) differentiation, as well as the expression of CDK1 and CDK2 (D) mRNA and (E) protein expression during HFT differentiation. Bands intensities were normalized to $\beta$-actin. Agomir-21 transfection blocks 1 ncRNA5322-mediated (F) proliferation and $(\mathrm{G})$ differentiation of HFTs. "P<0.05, ${ }^{* *} \mathrm{P}<0.01$. miR, microRNA; HFT, hair follicle stem cell; CDK, cyclin-dependent kinase; lnc, long non-coding; ns, not significant.

LncRNA AK015322 upregulates miR-21 expression and cell cycle during HFTs differentiation. miR-21 transfection has been reported to promote the differentiation of hair follicle-derived neural crest stem cells into Schwann cells (23). In the present study, it was demonstrated that miR-21 expression levels were upregulated by lncRNA5322 transfection-induced differentiation (Fig. 2A). The results revealed that lncRNA5322 transfection promotes CDK1 and CDK2 mRNA and protein expression in HFTs (Fig. 2B-D). These results suggest that LncRNA5322 upregulates miR-21 expression and increases CDK1 and CDK2 expression during HFT differentiation.

miR-21 transfection promotes HFT proliferation and differentiation. The role of miR-21 in the proliferation and differentiation of HFTs was investigated. Results demonstrated that miR-21 transfection (pmiR-21; Fig. 3A) promoted HFT proliferation and differentiation (Fig. 3B and C). Transfection with miR-21 also upregulated CDK1 and CDK2 mRNA and protein expression levels during HFTs differentiation (Fig. 3D and E). Agomir-21 transfection blocked lncRNA5322-mediated HFT proliferation and differentiation (Fig. 3F and G). These results indicate that transfection with miR-21 promotes HFT proliferation and differentiation and increases cyclin expression during HFT differentiation.

LncRNA AK015322 upregulates PI3K/AKT expression and phosphorylation during HFT differentiation. A previous study indicated that the PI3K/AKT signaling pathway is associated with stem cell differentiation (24). The association between IncRNA5322 and the PI3K/AKT signaling pathway during HFT differentiation was analyzed. The results revealed that lncRNA5322 transfection increased PI3K and 
A

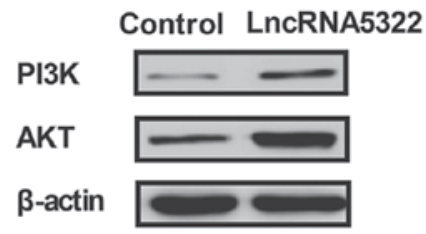

B
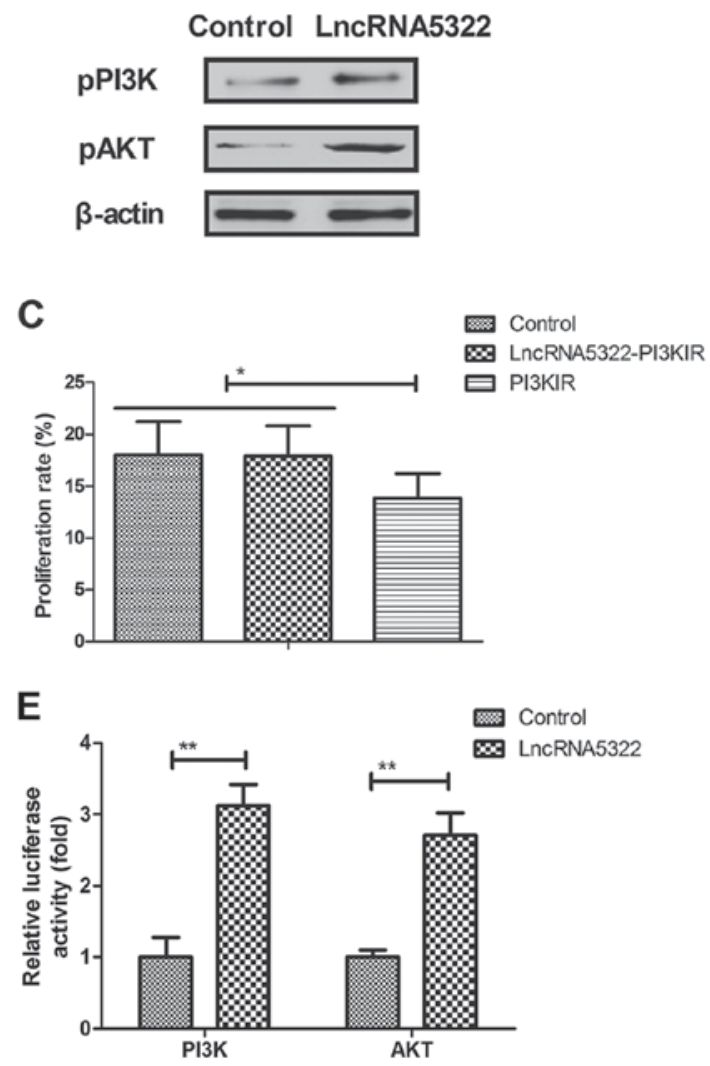
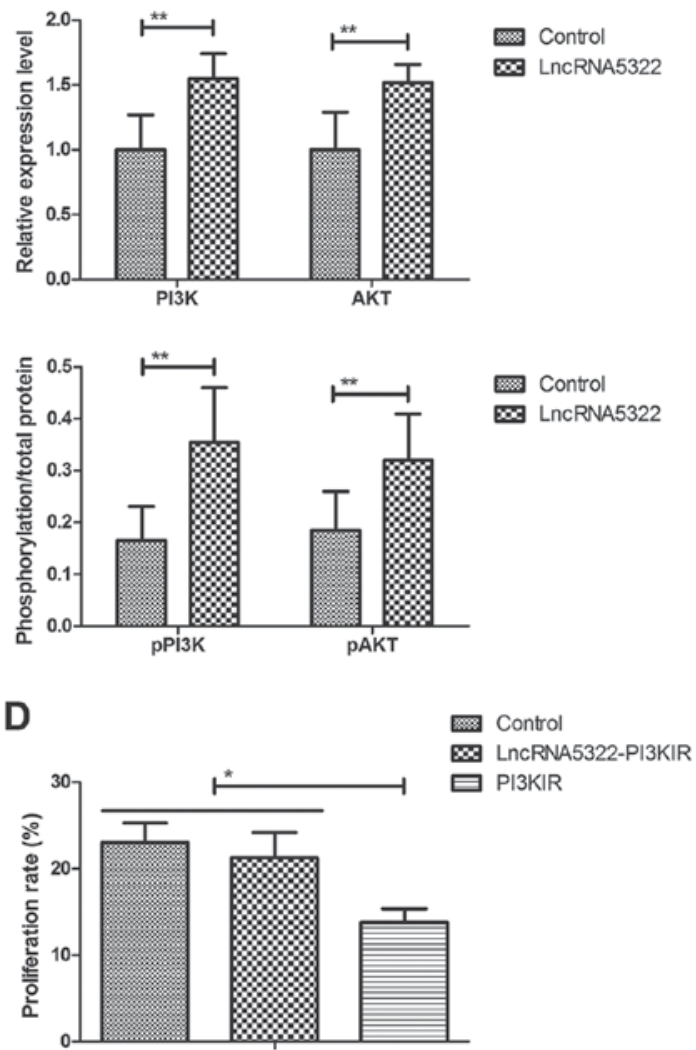

Figure 4. LncRNA5322 upregulates PI3K/AKT expression and phosphorylation during HFT differentiation. lncRNA5322 transfection increases PI3K and AKT (A) expression and (B) phosphorylation in HFTs. Bands intensities were normalized to $\beta$-actin. PI3K inhibitor inhibits lncRNA5322-induced (C) proliferation and (D) differentiation of HFTs. (E) LncRNA5322 transfection increases the luciferase activity of PI3K and AKT in HFTs ${ }^{*} \mathrm{P}<0.05$, ${ }^{* *} \mathrm{P}<0.01$. Lnc, long non-coding; PI3K, phosphoinositide-3-kinase; AKT, protein kinase B; HFT, hair follicle stem cell.

AKT expression and phosphorylation levels in HFTs (Fig. 4A and B). It was also demonstrated that PI3K inhibitor (PI3KIR) inhibited lncRNA5322-induced proliferation and differentiation of HFTs (Fig. 4C and D). Notably, the results of a luciferase gene report assay demonstrated that lncRNA5322 transfection increased the luciferase activity of PI3K and AKT (Fig. 4E). These results suggest that lncRNA5322 regulates proliferation and differentiation in HFTs via the PI3K/AKT signal pathway.

Agomir-21 blocks lncRNA AK015322-induced upregulation of the PI3K/AKT signaling pathway during HFT differentiation. The effects of agomir-21 on the PI3K/AKT signaling pathway in HFTs were investigated. It was demonstrated that agomir-21 transfection decreased PI3K and AKT expression and phosphorylation levels in HFTs (Fig. 5A and B). Agomir-21 transfection also blocked the lncRNA5322-induced upregulation of the PI3K/AKT signaling pathway during HFT differentiation (Fig. 5C and D).

\section{Discussion}

LncRNA has demonstrated a potential role in the progression of multilineage differentiation of HFTs $(25,26)$. The molecular characteristics and multipotency of HFT differentiation has been reviewed in bulge cells and dermal papilla mesenchyme cells as well as in the mechanism of hair growth (27). LncRNAs have been reported to be associated with mesenchymal stem cell differentiation via triple helix formation (28). The present study analyzed the regulatory effects of IncRNA5322 on HFT proliferation and differentiation and explored the potential mechanisms of the lncRNA5322-mediated signaling pathway. The results suggest that lncRNA5322 regulates HFT proliferation and differentiation via regulation of the miR-mediated PI3K/AKT signaling pathway.

A previous study regarding gene therapy and novel wound treatments reported that it is necessary to consider epidermal cells and HFTs as distinct populations (29). Hu et al (19) stated that IncRNA5322 is able to promote proliferation of C18-4 
A
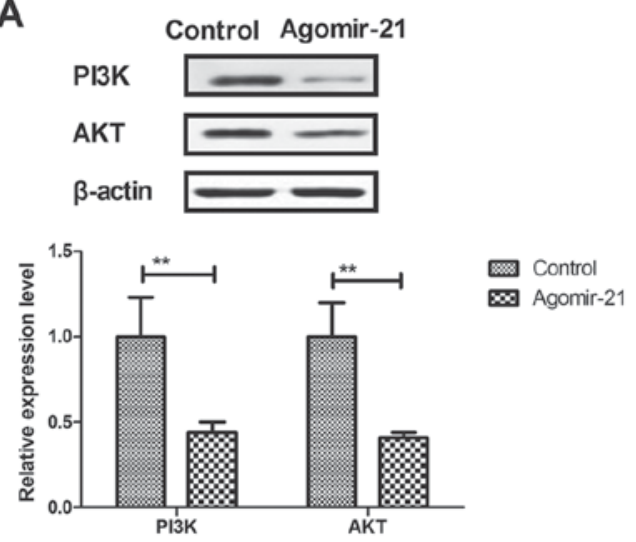

\section{C}
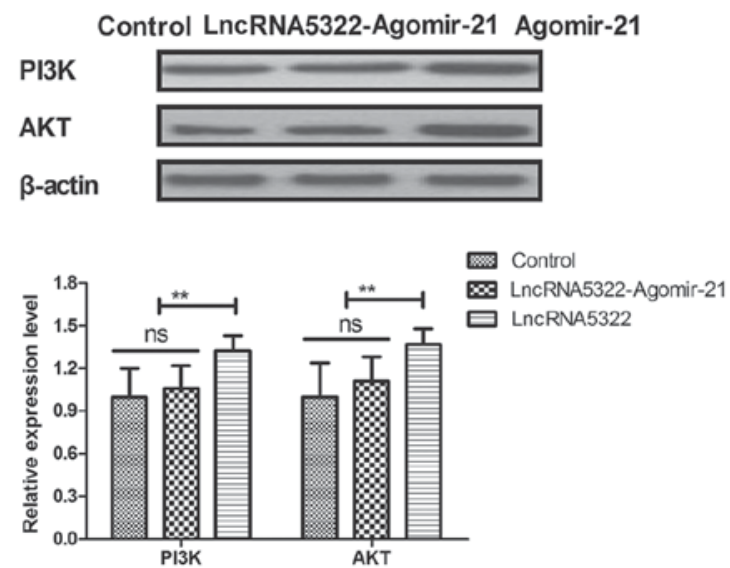

B

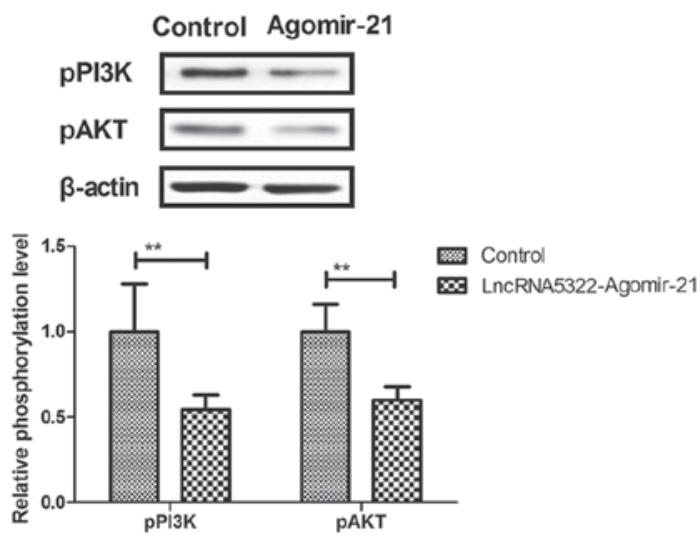

D
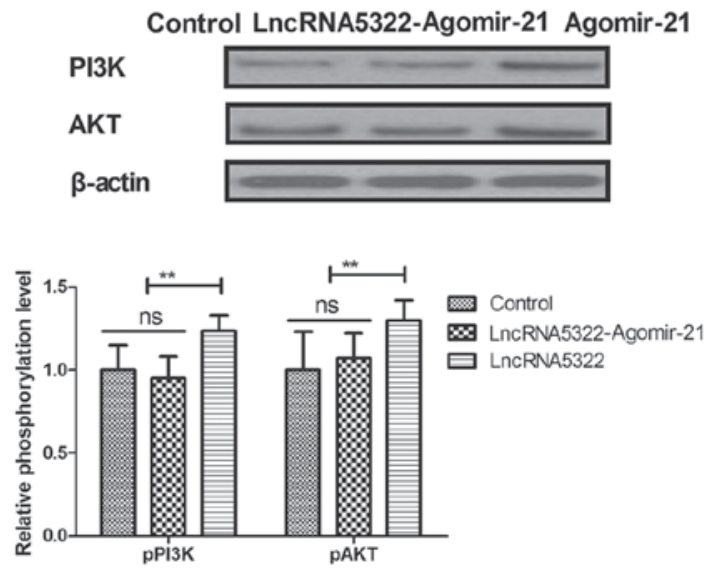

Figure 5. Agomir-21 blocks lncRNA5322-induced upregulation of the PI3K/AKT signaling pathway during HFT differentiation. Agomir-21 transfection decreases PI3K and AKT (A) expression and (B) phosphorylation in HFTs. Agomir-21 transfection blocks lncRNA5322-induced PI3K and AKT (C) expression and (D) phosphorylation during HFT differentiation. Bands intensities were normalized to $\beta$-actin. ${ }^{* *} \mathrm{P}<0.01$. Agomir-21, microRNA-21 agonist; PI3K, phosphoinositide-3-kinase; AKT, protein kinase B; Lnc, long non-coding; HFT, hair follicle stem cell.

spermatogonial stem cells by acting as a decoy for miR-19b-3p. In the present study, it was demonstrated that IncRNA5322 also stimulates the proliferation of and upregulates miR-21 expression in HFTs. Evidence has suggested that miR-21 promotes the differentiation of hair follicle-derived neural crest stem cells into Schwann cells (23). In the present study, it was observed that miR-21 transfection stimulates the proliferation and differentiation of HFTs, whereas agomir-21 blocks lncRNA5322-induced proliferation and differentiation. This suggests that lncRNA5322 may regulate proliferation and differentiation of HFTs via miR-21 expression.

To investigate the mechanism by which lncRNA5322 regulates the proliferation and differentiation of HFTs, the PI3K/AKT signaling pathway was assessed. The results demonstrated that the expression and phosphorylation of PI3K and AKT in 1ncRNA5322-transfected HFTs were significantly increased. A previous study reported that PI3K us able to regulate bone morphogenetic protein 2 -induced $\beta$-catenin activation in human bone marrow stem cells (30). A further study has indicated that the PI3K/AKT signaling pathway serves a critical role in neuron differentiation from human neural stem cells (31). The results of the present study demonstrate that lncRNA5322 or miR-21 transfection lead to upregulation of the PI3K/AKT signaling pathway in HFTs. Deng et al (32) suggested that miR-21 reduced hydrogen peroxide-induced apoptosis in c-kit+ cardiac stem cells in vitro via PTEN/PI3K/AKT signaling. The findings of the present study indicate that agomir-21 blocks lncRNA5322-induced upregulation of the PI3K/AKT signaling pathway during HFT differentiation. These results shed light on a potential novel signaling pathway responsible for lncRNA5322-mediated HFT differentiation.

The present study revealed that lncRNA5322 stimulates proliferation following $24 \mathrm{~h}$ transfection. However, cell cycle analysis was not performed following lncRNA5322 transfection in HFTs. Another limitation is that HFT differentiation was only analyzed using Oil Red O staining, not differentiation markers or fluorescence-activated cell sorting analysis. Additionally, previous studies have reported other potential molecular pathways that may be associated with the proliferation (33-37) and differentiation of HFTs, including the Wnt signal transduction pathway, forkhead box P1-mediated oxidative stress and epidermal growth factor receptor/extracellular signal-regulated kinases/AKT, c-Jun N-terminal kinases/c-Jun and TGF- $\beta$ pathways (38). The present study only investigated the PI3K/AKT signaling pathway in HFTs. Therefore, further experiments, including cell cycle analysis of HFTs and differentiation markers, are required to confirm the results of the present study. Other potential molecular pathways of HFT proliferation and differentiation should be considered. 
In conclusion, this study revealed that lncRNA5322 transfection promotes miR-21 expression and induces the proliferation and differentiation of HFTs via upregulating the PI3K/AKT signaling pathway. miR-21 is a direct target of lncRNA5322 in HFT differentiation, which provides a potential insight into the repair mechanism of injured skin by tissue engineering.

\section{References}

1. Jiang $\mathrm{C}$, Li X, Zhao $\mathrm{H}$ and Liu H: Long non-coding RNAs: Potential new biomarkers for predicting tumor invasion and metastasis. Mol Cancer 15: 62, 2016.

2. Bradford JR, Cox A, Bernard P and Camp NJ: Consensus analysis of whole transcriptome profiles from two breast cancer patient cohorts reveals long non-coding RNAs associated with intrinsic subtype and the tumour microenvironment. PLoS One 11: e0163238, 2016.

3. Tian X, Tian J, Tang X, Ma J and Wang S: Long non-coding RNAs in the regulation of myeloid cells. J Hematol Oncol 9: 99, 2016.

4. Etebari K, Asad S, Zhang G and Asgari S: Identification of aedes aegypti long intergenic non-coding RNAs and their association with wolbachia and dengue virus infection. PLoS Negl Trop Dis 10: e0005069, 2016.

5. Feng N, Ching T, Wang Y, Liu B, Lin H, Shi O, Zhang X, Zheng M, Zheng X, Gao M, et al: Analysis of microarray data on gene expression and methylation to identify long non-coding RNAs in non-small cell lung cancer. Sci Rep 6: 37233, 2016.

6. Fu Y, Biglia N, Wang Z, Shen Y, Risch HA, Lu L, Canuto EM, Jia W, Katsaros D and Yu H: Long non-coding RNAs, ASAP1-IT1, FAM215A, and LINC00472, in epithelial ovarian cancer. Gynecol Oncol 143: 642-649, 2016.

7. Gao Q, Ren H, Chen M, Niu Z, Tao H, Jia Y, Zhang J and Li W: Long non-coding RNAs regulate effects of $\beta$-crystallin B2 on mouse ovary development. Mol Med Rep 14: 4223-4231, 2016.

8. Guo JC, Li CQ, Wang QY, Zhao JM, Ding JY, Li EM and $\mathrm{Xu}$ LY: Protein-coding genes combined with long non-coding RNAs predict prognosis in esophageal squamous cell carcinoma patients as a novel clinical multi-dimensional signature. Mol Biosyst 12: 3467-3477, 2016

9. Hewson C, Capraro D, Burdach J, Whitaker N and Morris KV: Extracellular vesicle associated long non-coding RNAs functionally enhance cell viability. Noncoding RNA Res 1: 3-11, 2016.

10. Huang YK and Yu JC: Circulating microRNAs and long non-coding RNAs in gastric cancer diagnosis: An update and review. World J Gastroenterol 21: 9863-9886, 2015.

11. He R, Hu Z, Wang Q, Luo W, Li J, Duan L, Zhu YS and Luo DX: The role of long non-coding RNAs in nasopharyngeal carcinoma: As systemic review. Oncotarget 8: 16075-16083, 2017.

12. Shirai K, Hamada Y, Arakawa N, Yamazaki A, Tohgi N, Aki R, Mii S, Hoffman RM and Amoh Y: Hypoxia enhances differentiation of Hair Follicle-Associated-Pluripotent (HAP) stem cells to cardiac-muscle cells. J Cell Biochem 118: 554-558, 2017.

13. Minjuan W, Jun X, Shiyun S, Sha X, Haitao N, Yue W and Kaihong J: Hair follicle morphogenesis in the treatment of mouse full-thickness skin defects using composite human acellular amniotic membrane and adipose derived mesenchymal stem cells. Stem Cells Int 2016: 8281235, 2016.

14. Zhang Y, Xing Y, Guo H, Ma X and Li Y: Immunohistochemical study of hair follicle stem cells in regenerated hair follicles induced by Wnt10b. Int J Med Sci 13: 765-771, 2016.

15. Shen Q, Yu W, Fang Y, Yao M and Yang P: Beta-catenin can induce hair follicle stem cell differentiation into transit-amplifying cells through c-myc activation. Tissue Cell 49: 28-34, 2017.

16. Lien WH, Polak L, Lin M, Lay K, Zheng D and Fuchs E: In vivo transcriptional governance of hair follicle stem cells by canonical Wnt regulators. Nat Cell Biol 16: 179-190, 2014.

17. Wang Z, Pang L, Zhao H, Song L, Wang Y, Sun Q, Guo C, Wang B, Qin X and Pan A: miR-128 regulates differentiation of hair follicle mesenchymal stem cells into smooth muscle cells by targeting SMAD2. Acta Histochem 118: 393-400, 2016

18. Zou ZW, Ma C, Medoro L, Chen L, Wang B, Gupta R, Liu T, Yang XZ, Chen TT, Wang RZ, et al: LncRNA ANRIL is up-regulated in nasopharyngeal carcinoma and promotes the cancer progression via increasing proliferation, reprograming cell glucose metabolism and inducing side-population stem-like cancer cells. Oncotarget 7: 61741-61754, 2016.
19. Hu K, Zhang J and Liang M: LncRNA AK015322 promotes proliferation of spermatogonial stem cell C18-4 by acting as a decoy for microRNA-19b-3p. In Vitro Cell Dev Biol Anim 53: 277-284, 2017.

20. Yabut O, Domogauer J and D'Arcangelo G: Dyrk1A overexpression inhibits proliferation and induces premature neuronal differentiation of neural progenitor cells. J Neurosci 30: 4004-4014, 2010.

21. Liu D, Yi C, Zhang D, Zhang J and Yang M: Inhibition of proliferation and differentiation of mesenchymal stem cells by carboxylated carbon nanotubes. ACS Nano 4: 2185-2195, 2010.

22. Livak KJ and Schmittgen TD: Analysis of relative gene expression data using real-time quantitative PCR and the 2(-Delta Delta C(T)) method. Methods 25: 402-408, 2001

23. Ni Y, Zhang K, Liu X, Yang T, Wang B, Fu L, A L and Zhou Y: miR-21 promotes the differentiation of hair follicle-derived neural crest stem cells into Schwann cells. Neural Regen Res 9: 828-836, 2014.

24. Cui J, Zhang F, Wang Y, Liu J, Ming X, Hou J, Lv B, Fang S and Yu B: Macrophage migration inhibitory factor promotes cardiac stem cell proliferation and endothelial differentiation through the activation of the PI3K/Akt/mTOR and AMPK pathways. Int $\mathrm{J}$ Mol Med 37: 1299-1309, 2016.

25. Sieber-Blum M and Grim M: The adult hair follicle: Cradle for pluripotent neural crest stem cells. Birth Defects Res C Embryo Today 72: 162-172, 2004.

26. Osawa $M$ and Nishimura EK: Stem cells in the mammalian hair follicle. Tanpakushitsu Kakusan Koso 49: 727-733, 2004 (In Japanese).

27. Ma DR, Yang EN and Lee ST: A review: The location, molecular characterisation and multipotency of hair follicle epidermal stem cells. Ann Acad Med Singapore 33: 784-788, 2004.

28. Kalwa M, Hänzelmann S, Otto S, Kuo CC, Franzen J, Joussen S, Fernandez-Rebollo E, Rath B, Koch C, Hofmann A, et al: The lncRNA HOTAIR impacts on mesenchymal stem cells via triple helix formation. Nucleic Acids Res 44: 10631-10643, 2016.

29. Ito M, Liu Y, Yang Z, Nguyen J, Liang F, Morris RJ and Cotsarelis G: Stem cells in the hair follicle bulge contribute to wound repair but not to homeostasis of the epidermis. Nat Med 11: $1351-1354,2005$.

30. Lee JH, Kim BG, Ahn JM, Park HJ, Park SK, Yoo JS, Yates JR III and Cho JY: Role of PI3K on the regulation of BMP2-induced beta-Catenin activation in human bone marrow stem cells. Bone 46: 1522-1532, 2010.

31. Ojeda L, Gao J, Hooten KG, Wang E, Thonhoff JR, Dunn TJ, Gao T and Wu P: Critical role of PI3K/Akt/GSK3 $\beta$ in motoneuron specification from human neural stem cells in response to FGF2 and EGF. PLoS One 6: e23414, 2011.

32. Deng W, Wang Y, Long X, Zhao R, Wang Z, Liu Z, Cao S and Shi B: miR-21 reduces hydrogen peroxide-induced apoptosis in c-kit+ cardiac stem cells in vitro through PTEN/PI3K/Akt signaling. Oxid Med Cell Longev 2016: 5389181, 2016.

33. Shao Y, Ni Z and Li Y: Wnt signal transduction pathways and hair follicle stem cells. Sheng Wu Yi Xue Gong Cheng Xue Za Zhi 27: 945-948, 2010 (In Chinese).

34. Zhao J, Li H, Zhou R, Ma G, Dekker JD, Tucker HO, Yao Z and Guo X: Foxp1 regulates the proliferation of hair follicle stem cells in response to oxidative stress during hair cycling. PLoS One 10: e0131674, 2015.

35. Bai T, Liu F, Zou F, Zhao G, Jiang Y, Liu L, Shi J, Hao D, Zhang Q, Zheng T, et al: Epidermal growth factor induces proliferation of hair follicle-derived mesenchymal stem cells through epidermal growth factor receptor-mediated activation of ERK and AKT signaling pathways associated with upregulation of cyclin D1 and downregulation of p16. Stem Cells Dev 26: 113-122, 2017

36. Liu X, Song L, Liu J, Wang S, Tan X, Bai X, Bai T, Wang Y, Li M, Song Y and Li Y: miR-18b inhibits TGF- $\beta 1$-induced differentiation of hair follicle stem cells into smooth muscle cells by targeting SMAD2. Biochem Biophys Res Commun 438: 551-556, 2013.

37. Sarate RM, Chovatiya GL, Ravi V, Khade B, Gupta S and Waghmare SK: sPLA2-IIA overexpression in mice epidermis depletes hair follicle stem cells and induces differentiation mediated through enhanced JNK/c-Jun activation. Stem Cells 34: 2407-2417, 2016.

38. Yeh YH, Wang SW, Yeh YC, Hsiao HF and Li TK: Rhapontigenin inhibits TGF- $\beta$-mediated epithelial-mesenchymal transition via the PI3K/AKT/mTOR pathway and is not associated with HIF-1 $\alpha$ degradation. Oncol Rep 35: 2887-2895, 2016. 